\title{
Ecología trófica del jabalí europeo (Sus scrofa) silvestre en Chile
}

\author{
Trophic ecology of the wild boar (Sus scrofa) in Chile
}

ÓSCAR SKEWES ${ }^{1}$, ROBERTO RODRÍGUEZ ${ }^{2} \&$ FABIÁN M. JAKSIC ${ }^{3}$

\author{
${ }^{1}$ Departamento de Ciencias Pecuarias, Facultad de Medicina Veterinaria, Universidad de Concepción, Chile; \\ e-mail: oskewes@udec.cl \\ ${ }^{2}$ Departamento de Botánica, Facultad de Ciencias Naturales y Oceanográficas, Universidad de Concepción, Chile \\ ${ }^{3}$ Centro de Estudios Avanzados en Ecología \& Biodiversidad (CASEB), Pontificia Universidad Católica de Chile, \\ Santiago, Chile; e-mail: fjaksic@bio.puc.cl
}

\begin{abstract}
RESUMEN
Documentamos la dieta del jabalí silvestre en el centro-sur de Chile basados en el examen de 20 estómagos colectados en los faldeos de volcán Mocho-Choshuenco ( $39^{\circ} 54^{\prime} \mathrm{S}, 72^{\circ} 02^{\prime} \mathrm{O}$ ) y en el Parque Nacional Vicente Pérez Rosales $\left(41^{\circ} 03^{\prime} \mathrm{S}, 71^{\circ} 54^{\prime} \mathrm{O}\right)$. La dieta está representada por animales, vegetales y hongos. Entre los vegetales y hongos son consumidas tanto las partes epígeas como hipógeas, lo mismo que frutos y semillas. Hongos y rizomas de Gunnera tinctoria constituyen ítemes vegetales cuantitativamente importantes en la alimentación. El jabalí forrajea en general en ambiente boscoso, pero las especies vegetales más frecuentes en la dieta se encuentran en espacios abiertos o fuera del bosque. El ambiente dominado por Chusquea spp. constituye un importante sitio de alimentación. Los roedores de la familia Muridae dominan entre los animales determinados en la dieta del jabalí. Esta situación, sin embargo, parece obedecer a la ocurrencia de una ratada producto de la semillación masiva de Chusquea spp. durante la época de muestreo. Las aves de la familia Rhinocryptidae e invertebrados (en especial larvas del coleóptero Chiasognathus grantii) constituyen parte frecuente de los ítemes animales de la dieta del jabalí. En comparación a estudios en otras partes del mundo, el jabalí silvestre en Chile consume hongos en porcentaje de ocurrencia similar (65\%) a su dieta en Estados Unidos de América pero bastante más alta que su dieta en Europa (5-32 \%). En relación al volumen del componente animal, el jabalí en Chile consume más (16,1\%) que en Estados Unidos de América (6\%) y que en Europa (7-13\%).
\end{abstract}

Palabras clave: especie invasora, Sudamérica, bosque templado, ratada, semillación masiva.

\begin{abstract}
We document the diet of wild boar in south-central Chile based on analysis of 20 stomachs collected on the outskirts of Mocho-Choshuenco volcano $\left(39^{\circ} 54^{\prime} \mathrm{S}, 72^{\circ} 02^{\prime} \mathrm{W}\right.$ ) and of Vicente Pérez Rosales National Park ( $\left.41^{\circ} 03^{\prime} \mathrm{S}, 71^{\circ} 54^{\prime} \mathrm{W}\right)$. The diet incorporates animals, plants and fungi. Among the latter two, both epigeal and hypogeal parts are consumed, together with fruits and seeds. Fungi and rhizomes of Gunnera tinctoria are the most abundant plant items in the diet. The wild boar in Chile forages chiefly in forested environments, but nonetheless the most frequent plant items in its diet are found in open spaces or outside the forest. Environments dominated by bamboos Chusquea spp. constitute important foraging sites. Rodents in the family Muridae prevail among animal items in the wild boar diet. This situation, however, is associated to the occurrence of a mouse outbreak (ratada) resulting from the mast seeding of Chusquea spp. during our study. Birds in the family Rhinocryptidae together with invertebrates (specially Coleopteran larvae of Chiasognathus grantii) made up a sizeable part of the animals in the wild boar diet. In comparison to elsewhere, the wild boar in Chile consumes fungi in a similar percentage of occurence (65\%) as in the United States of America but much higher than in Europe (5-32\%). With regard to the animal component, the wild boar in Chile consumes more (20\% volume) of such items than in the United States of America $(6 \%)$ and in Europe (7-13\%).
\end{abstract}

Key words: invasive species, South America, temperate forest, mouse outbreak, mast seeding.

\section{INTRODUCCIÓN}

Las especies introducidas o exóticas se consideran como un factor importante en la alteración de la biodiversidad (Vitousek et al.
1997), modificando dinámicas comunitarias, tramas tróficas y procesos ecosistémicos, con resultados económicos generalmente negativos (Mooney et al. 2005). Entre las especies invasoras más desconocidas en Chile está el 
jabalí (Sus scrofa Linnaeus, 1758), un animal con enorme área de distribución original en el continente euroasiático, que incluye hábitats muy diversos (Genov 1981a), y que ha tenido éxito en establecerse en todos los continentes donde ha sido introducido (Lever 1985, Long 2003).

El juicio acerca del impacto de Sus scrofa ya sea como jabalí o como cerdo asilvestrado sobre la flora y fauna en todas las áreas donde se ha establecido (Argentina, Australia, Estados Unidos de América, Hawaii, Islas Galápagos, Nueva Zelandia), es negativo. En la flora se mencionan modificaciones de la composición de especies, extinción local de plantas, reducción de la diversidad, alteración de la cubierta del suelo que facilita la colonización de plantas exóticas. La fauna a su vez, es afectada por depredación, destrucción de nidos, competencia alimentaria y destrucción de hábitat (Wodzicki 1950, Howard 1964, Bratton 1975, Challies 1975, Wood \& Roark 1980, Howe et al. 1981, Singer 1981, Tisdell 1982, 1984; Alexiou 1983, Campbell \& Rudge 1984, Coblentz \& Baber 1987, Vtrorov 1993, Lever 1994, Welander 1995, Long 2003, Cruz et al. 2005). La presencia del jabalí europeo en Chile como especie exótica ha sido mencionada por diversos autores (Mann 1973, Petrides 1975, Miller \& Rottmann 1976, Daciuk 1978, Pine et al. 1979, Tisdell 1982, Heck \& Raschke 1985, Skewes 1990, Jaksic 1998, Jaksic et al. 2002, Mella et al. 2002, Long 2003). Sin embargo, sus efectos sobre la fauna y flora nativas son casi desconocidos (Jaksic 1998), con la excepción de los aportes contenidos en una tesis doctoral (Skewes 1990).

El efecto o impacto de una especie invasora puede en gran medida inferirse de su posición trófica en las comunidades, a través de analizar su dieta. Frutos y semillas son de gran importancia en la dieta del jabalí en Europa, Asia y costa del mar de Japón. Semillas de árboles de los géneros Quercus y Fagus, especialmente cuando ocurre semillación masiva, desempeñan un extraordinario papel en la biología del jabalí tanto en Eurasia (Oloff 1951, Briedermann 1976, Genov 1981b, Groot Bruinderink et al. 1994, Bieber \& Ruf 2005) como en Norteamérica (Henry \& Conley1972). Ninguno de estos géneros arbóreos está presente en Chile en forma silvestre. Cultivos agrícolas tales como papas, cereales y maíz representan una importante parte de la dieta del jabalí en Europa (Mackin 1970, Genov 1981c, Briedermann 1986, Schley \& Roper 2003, Herrero et al. 2006), Estados Unidos de América (Barret 1977, Everitt \& Alaniz 1980), Argentina (Anónimo 1956, Consejo Federal de Inversiones 1961) y también para los cerdos asilvestrados en Australia (Tisdell 1982) y en Nueva Zelandia (Wodzicki 1950, Challies 1975). En Chile, estos cultivos no se encuentran en el área de distribución del jabalí, excepto por pequeñas superficies para consumo doméstico.

El jabalí es una especie oportunista omnívora cuya dieta está determinada por la abundancia relativa de diferentes tipos de alimentos y de material vegetal rico en energía (Schley \& Roper 2003). Sin embargo, no se dispone de datos de dieta del jabalí europeo en Sudamérica como especie silvestre exótica (Lever 1985, Long 2003). Los antecedentes expuestos nos permiten proponer que el jabalí usará en Chile una amplia gama de recursos tróficos vegetales y animales locales. Nuestro trabajo aporta al conocimiento de la dieta del jabalí en bosques templados andinos de Chile y permite hacer inferencias sobre su posición trófica en este país, lo cual puede contribuir a determinar su impacto sobre la flora y fauna nativas.

\section{MATERIALES Y MÉTODOS}

\section{Sitios de estudio}

Las muestras y observaciones de terreno provienen de capturas en un área de cerca de 50.000 ha que cubre los faldeos este y oeste del volcán Mocho-Choshuenco (39 54' S, $72^{\circ} 02^{\prime}$ $\mathrm{O}$, codificado como $\mathrm{VMCH}$ ) y de $136 \mathrm{~km}$ al sur en el sector Casa Pangue $\left(41^{\circ} 03^{\prime} \mathrm{S}, 71^{\circ} 54^{\prime} \mathrm{O}\right)$, abarcando cerca de 5.000 ha del Parque Nacional Vicente Pérez Rosales (codificado como PNVPR). Climáticamente, las dos áreas en general corresponden a la región oceánica con influencia mediterránea. Edáficamente, hay suelos derivados de cenizas volcánicas y en gran parte podzol. Vegetacionalmente, la región está poblada por selva valdiviana andina, microtérmica, con un estrato herbáceo reducido. Topográficamente, presenta valles estrechos con fuerte pendiente, dominados por 
bosques (Armesto et al. 1997). Las estaciones meteorológicas más cercanas indican una temperatura media de $14,2{ }^{\circ} \mathrm{C}$ y precipitación anual de $2.402 \mathrm{~mm}$ anuales (Estación Loncoche) para $\mathrm{VMCH}$ y de $11,1{ }^{\circ} \mathrm{C}$ y 2.342 mm anuales (Estación Puerto Montt) en PNVPR (Hajek \& di Castri 1975).

En términos florísticos, el sector $\mathrm{VMCH}$ presenta el tipo forestal Coigüe-Raulí-Tepa (Nothofagus dombeyi, N.alpina y Laurelia philippiana) y Roble-Raulí-Coihue (N. obliqua$N$. alpina- $N$. dombeyi) (sensu Donoso 1981). En PNVPR domina el tipo forestal CoihueRaulí-Tepa acompañado de Siempreverde (sensu Donoso1981) caracterizado por árboles perennifolios en diferentes combinaciones (Donoso 1993) y que incluyen a $N$. dombeyi, Eucryphia cordifolia, Aextoxicon punctatum, Saxegothaea conspicua, Weinmannia trichosperma junto a Amomyrtus luma, Lomatia ferruginea, Dasyphyllum diacanthoides y en algunos sectores Gevuina avellana. Veblen et al. (1989) describen en detalle la flora del valle donde se capturaron los jabalíes en PNVPR.

Recolección, procesamiento y análisis de muestras

Se recolectó el contenido estomacal de 20 jabalíes, incluyendo 15 adultos (> 24 meses) y 5 subadultos o juveniles (1 a 2 años), edades estimadas de acuerdo a Matschke (1967) y Briederman (1986). Doce ejemplares, compuestos por 10 adultos (siete machos y tres hembras) y dos subadultos (dos hembras) fueron cazados entre los años 1986 y 1996 en los faldeos del volcán Mocho-Choshuenco y ocho muestras de cinco animales adultos (dos hembras y tres machos) y tres juveniles (dos machos y una hembra) fueron recolectadas en invierno de 1995 de animales del sector Casa Pangue. Las muestras se tomaron luego de abatir el animal, abriendo la curvatura mayor del estómago y muestreando porciones de contenido del estómago hasta completar 500 $\mathrm{mL}$ (el contenido estomacal de los jabalíes examinados comprendía entre 1,5 y $3,3 \mathrm{~kg}$ ). El material fue almacenado en frascos con abundante solución fijadora AFA (90\% alcohol $70^{\circ}, 5 \%$ formalina y $5 \%$ ácido acético). El análisis de contenido estomacal siguió la metodología indicada por Wood \& Roark (1980) modificada por Skewes (1990). La caza en el parque fue autorizada por CONAF X Región y mediante resolución Exenta $\mathrm{N}^{\circ} 256$ del 26.01.1995 del Servicio Agrícola y Ganadero. Las 20 muestras analizadas en este trabajo se obtuvieron en las siguientes épocas: cinco en otoño; once en invierno, dos en primavera y dos en verano.

Los organismos encontrados en el estómago fueron identificados al microscopio y lupa estereoscópica. El estado de destrucción de algunos ítemes dificultó su reconocimiento, y en algunos casos se determinó por fracciones de ellos, usando el nivel taxonómico más fino posible. La identificación de los ítemes la realizaron los investigadores junto a especialistas de los departamentos de Botánica y de Zoología de la Universidad de Concepción, para lo cual se contó con colecciones del herbario y ejemplares del museo almacenados en la Facultad de Ciencias Naturales y Oceanográficas de la misma institución, como también con la colección de referencia recolectada en terreno. La clasificación taxonómica siguió a Marticorena \& Quezada (1985) para plantas, a Tamayo et al. (1987) para mamíferos, a Peña (1988) para invertebrados y a Garrido (1988) para hongos .

Se utilizaron dos indicadores tróficos: (a) la frecuencia de ocurrencia (\%) se calculó multiplicando por 100 el número de estómagos con un determinado ítem alimentario y luego dividiendo por el número total de estómagos examinados; (b) el porcentaje volumétrico (\% vol) de cada ítem alimentario (>1 mL) se midió en probeta graduada por desplazamiento de agua, calculando el porcentaje de volumen sobre el volumen total de cada ítem registrado en la dieta, dividido por el total del volumen de ítemes identificados en los estómagos.

Debido a la gran cantidad de restos de rizoma de $G$. tinctoria encontrados en los contenidos estomacales de las muestras, se analizó su composición mediante análisis químico proximal en el Laboratorio de Bromatología de la Facultad de Farmacia y en el de Nutrición Animal de la Facultad de Agronomía de la Universidad de Concepción. Se analizaron rizomas $(0,5-1 \mathrm{~kg}$ de materia verde) colectados cuatro en mayo de 1989 en la zona de VMCH y cinco en julio de 2001 en PNVPR. El análisis químico proximal se basó en el método de la AOAC (1997) para medir humedad, proteína Bruta (PB), extracto etéreo 
(EE), cenizas (C), y fibra bruta (FB). El extracto no nitrogenado se obtuvo restando de 100 , los porcentajes de humedad, proteína bruta, grasa bruta, cenizas y fibra bruta. Para predecir el valor energético (ED) se usó la fórmula para cerdos de $\mathrm{ED}\left(\mathrm{MJ} \mathrm{kg} \mathrm{k}^{-1}\right)=$ $17,38+0.0105 \mathrm{~PB}+0.0114 \mathrm{EE}-0.031 \mathrm{FB}-0.0402 \mathrm{C}$ (McDonald et al. 1993).

Para comparar la composición volumétrica de los contenidos entre ambas localidades, se agruparon los ítemes con mayor representación en la dieta en categorías tales como: Vegetales (total de ítemes vegetales incluyendo hongos), Gunnera tinctoria, Hongos, Otros vegetales (total de vegetales menos G. tinctoria menos hongos), Animales (total de vertebrados más invertebrados), Vertebrados e Invertebrados (Tabla 3). Estos datos de volumen fueron analizados mediante la prueba $t$ de Student para muestras independientes con prueba de homogeneidad de varianzas tanto entre localidades, como para efecto sexo y edad en programa Systat, v.10.

\section{RESULTADOS}

\section{Especies vegetales en la dieta en dos localidades}

Se identificaron 25 especies de plantas vasculares y siete de hongos como parte de la dieta del jabalí (Tabla 1). Entre las especies vegetales resalta la alta frecuencia de rizomas de la nalca Gunnera tinctoria, que aparecen en 18 de los 20 estómagos examinados $(90 \%)$ y también por su aporte volumétrico con 15,2\% (Tabla 3). En terreno, durante el invierno era muy frecuente observar rizomas de nalca roídos por jabalí. En un estómago se encontró un pecíolo de nalca, solo en otoño. El análisis químico proximal de rizoma de nalca reveló los siguientes valores promedio en porcentaje base seca: PB: 7,5; EE: 2,0; FB:10,8; C: 8,6 y extracto no nitrogenado de $73,7 \%$ con una ED estimada de $17,1 \mathrm{MJ} \mathrm{kg}^{-1} \mathrm{MS}$.

Las brácteas de quila Chusquea spp. se detectaron con regularidad en las muestras (en 15 de $20=75 \%$, Tabla 1), pero en bajo volumen. En dos estómagos (VMCH en verano) se registraron gran cantidad $(38,7 \%$ vol $)$ de restos de tallos o culmos de esta gramínea. Los culmos emergen de noviembre a febrero dependiendo de las condiciones de luz del sitio y son consumidos por el jabalí cuando están en estado tierno (O. Skewes resultados no publicados). En terreno era frecuente observar abundante remoción de suelo que hace el jabalí mediante el morro (acción de hozar), bajo matorrales de quila.

Los hongos son otro ítem que destaca por su alta presencia (65\%, Tabla 1) en los estómagos, detectándose numerosos cuerpos fructíferos hipogeos, esféricos de color pardo, de $1-3 \mathrm{~cm}$ de diámetro, de difícil clasificación por el escaso conocimiento disponible al respecto.

Las hojas de gramíneas (distintas de bambúseas) son monocotiledónea y dicotiledóneas sobre las cuales no se pudo avanzar más en su identificación. Ellas se detectan con alta frecuencia en las muestras (75 $\%$, Tabla 1) (Tabla 3). Las hojas de Nothofagus dombeyi se encontraron en la mitad de las muestras pero en escasa cantidad (Tabla 1). Cortaderia sp. se presenta tanto en estómagos de VMCH como de PNVPR con alta regularidad (Tabla 1) pero en cantidades discretas. Una jabalina adulta en PNVPR mostró gran cantidad (> $40 \%$ vol) de esta gramínea en su contenido estomacal.

Por otra parte, los rizomas y hojas del helecho costilla de vaca (Blechnum chilense) están representados en un tercio de las muestras de estómago de PNVPR (invierno). En terreno se verificó el consumo del rizoma de esta especie en verano y en algunos casos la hozadura implicaba el desarraigamiento de la planta. En las muestras de VMCH no se detectó la presencia del helecho B. chilense. Raíz de la orquídea Chloraea gaudichaudii se registró en solo dos muestras de invierno en PNVPR (Tabla 1), pero en terreno era común encontrar el consumo de rizoma de esta planta durante el otoño. Restos de frutos del avellano Gevuina avellana se constataron en el contenido estomacal de jabalíes capturados tanto en VMCH como en PNVPR (Tabla 1). Además, en terreno y en época de otoño, era frecuente observar restos de cáscara del fruto partidos por la mitad, típico del consumo por jabalí.

Aquellas especies vegetales que crecen o se encuentran habitualmente en el bosque y quebradas comprometen $76 \%$ del total de los ítemes vegetales detectados (Tabla 1). En tanto que aquellas especies que se encuentran en lugares abiertos, fuera del bosque, aportan $24 \%$ de los ítemes (Tabla 1). 


\section{TABLA 1}

Ítemes vegetales en la dieta del jabalí silvestre en Chile, basado en estómagos de dos localidades, porcentaje de ocurrencia en el total de estómagos y hábitat de la especie vegetal consumida.

Plant items in the diet of wild boar in Chile, based on stomachs by locality, percent occurrence across stomachs, and habitat of the plant species eaten.

\begin{tabular}{|c|c|c|c|c|}
\hline \multirow[t]{2}{*}{ Ítemes vegetales } & \multicolumn{2}{|c|}{ Detección en estómagos } & \multirow[t]{2}{*}{ Ocurrencia total (\%) } & \multirow[t]{2}{*}{ Hábitat } \\
\hline & $\begin{array}{c}\text { Volcán Mocho- } \\
\text { Choshuenco }(n=12)\end{array}$ & $\begin{array}{c}\text { Parque Nacional } \\
\text { Vicente Pérez Rosales } \\
(\mathrm{n}=8)\end{array}$ & & \\
\hline \multicolumn{5}{|l|}{ Hojas } \\
\hline Blechnum chilense & & + & 30,0 & Quebrada $^{\mathrm{a}}$ \\
\hline Chusquea sp. & + & + & 75,0 & Bosque \\
\hline Cortaderia sp. & + & + & 40,0 & Abierto $^{\mathrm{b}}$ \\
\hline Cyperus sp. & + & & 5,0 & Humedal ${ }^{\mathrm{c}}$ \\
\hline Hypochaeris radicata & + & & 15,0 & Bosque \\
\hline Juncus sp. & + & & 10,0 & Abierto $^{\mathrm{b}}$ \\
\hline Mimulus luteus & + & & 5,0 & Quebrada $^{\mathrm{a}}$ \\
\hline Myrceugenia sp. & & + & 10,0 & Bosque \\
\hline Nothofagus dombeyi & + & + & 50,0 & Bosque \\
\hline Osmorhiza chilensis & + & & 5,0 & Bosque \\
\hline Ribes sp. & + & & 5,0 & Bosque \\
\hline Saxegothaea conspicua & & + & 5,0 & Bosque \\
\hline Pseudotsuga menziensii* ${ }^{*}$ & + & & 5,0 & Bosque \\
\hline Sphagnum sp. & + & & 5,0 & Bosque \\
\hline $\begin{array}{l}\text { Gramíneas spp. } \\
\text { Pecíolo }\end{array}$ & + & + & 75,0 & Abierto $^{\mathrm{b}}$ \\
\hline $\begin{array}{l}\text { Gunnera tinctoria } \\
\text { Tallos }\end{array}$ & + & & 5,0 & Abierto $^{\mathrm{b}}$ \\
\hline Chusque a couleu & + & & 10,0 & Bosque \\
\hline Luzuriaga radicans & & + & 5,0 & Bosque \\
\hline \multicolumn{5}{|l|}{ Semillas o frutos } \\
\hline Fuchsia megellanica & + & & 5,0 & Bosque \\
\hline Gevuina avellana & + & + & 10,0 & Bosque \\
\hline Greigia sphacelata & + & & 5,0 & Bosque \\
\hline Gunnera tinctoria & + & & 5,0 & Abierto $^{\mathrm{b}}$ \\
\hline Lapageria rosea & + & & 5,0 & Bosque \\
\hline Ribes sp. & + & & 5,0 & Bosque \\
\hline Ugni molinae & + & & 5,0 & Abierto $^{b}$ \\
\hline \multicolumn{5}{|l|}{ Raíces } \\
\hline Hypochaeris radicata & + & + & 20.0 & Bosque \\
\hline Osmorhiza chilensis & & + & 5.0 & Bosque \\
\hline Chloraea gaudichaudii & & + & 10.0 & Abierto $^{\mathrm{b}}$ \\
\hline Gunnera tinctoria & + & + & 5.0 & Abierto $^{\mathrm{b}}$ \\
\hline Luzuriaga radicans & & + & 5.0 & Bosque \\
\hline Gramíneas spp. & + & + & 45.0 & Abierto $^{\mathrm{b}}$ \\
\hline Juncáceas spp. & & + & 5.0 & Humedal $^{\mathrm{c}}$ \\
\hline Dicotiledóneas spp. & & + & 5.0 & \\
\hline \multicolumn{5}{|l|}{ Rizomas } \\
\hline Blechnum chilense & & + & 5.0 & Quebrada $^{\mathrm{a}}$ \\
\hline Gunnera tinctoria & + & + & 90.0 & Abierto $^{\mathrm{b}}$ \\
\hline Osmorhiza chilensis & + & & 5.0 & Bosque \\
\hline \multicolumn{5}{|l|}{ Hongos } \\
\hline Agaricus spp. & + & & & Bosque \\
\hline Aphiluphoral sp. & + & & & Bosque \\
\hline Citaria sp. & + & & & Bosque \\
\hline Cortinarius spp. & + & & & Bosque \\
\hline Hymenogaster sp. & + & & & Bosque \\
\hline Hysterangium purpureum & + & & & Bosque \\
\hline Paxillus spp. & + & & & Bosque \\
\hline Cuerpos fructíferos e hifas & + & + & 70.0 & Bosque \\
\hline
\end{tabular}

+ Presencia detectada; * especie exótica; a quebrada o curso de agua; ${ }^{\text {a }}$ abierto, fuera del bosque; ${ }^{\mathrm{c}}$ humedal abierto 
En cuanto al aporte volumétrico (Tabla 3 ) el ítem "Otros vegetales" compuesto principalmente por gramíneas y que no incluye hongos ni Gunnera, es el más representativo de los componentes vegetales de la dieta con valor promedio de $42,0 \%$ de volumen y extremos de 0,5 a $83,6 \%$ (Tabla 3). Hongos y la nalca Gunnera tinctoria le siguen con valores promedio de 15,9 y $15,2 \%$ respectivamente. En hongos se verificaron extremos de 0,1 a 76,5\% vol y en nalca de 3,6 a 82,6\% vol (Tabla 3). Los mayores volúmenes de consumo de hongos se presentaron en muestras de otoño y los de nalca en invierno. Otro ítem destacado por su aporte en volumen es animales con 16,1\% vol.

Especies animales en la dieta en dos localidades

Entre las especies animales presentes en el contenido estomacal de jabalí sobresale por ocurrencia la clase Insecta con $70 \%$, luego siguen Mammalia y Aves con igual proporción (45\%) y más atrás Amphibia (25\%) (Tabla 2).

TABLA 2

Ítemes animales por porcentaje de ocurrencia en contenidos estomacales de jabalí en dos localidades de Chile

Animal items as percent occurrence by locality in stomach contents of wild boar in Chile

\begin{tabular}{|c|c|c|}
\hline Ítems animales & $\begin{array}{l}\text { Volcán Mocho-Choshuenco } \\
\qquad(\mathrm{n}=12)\end{array}$ & $\begin{array}{l}\text { Parque Nacional Vicente Pérez Rosales } \\
\qquad(\mathrm{n}=8)\end{array}$ \\
\hline \multicolumn{3}{|l|}{ Mammalia: Muridae } \\
\hline Abrothrix olivaceus & & 100,0 \\
\hline Geoxus valdivianus & 8,3 & \\
\hline Oligoryzomys longicaudatus & & 100,0 \\
\hline \multicolumn{3}{|l|}{ Aves: Rhinocryptidae } \\
\hline Scelorchilus rubecula & 16,7 & 62,5 \\
\hline Pteroptochos tarnii & 8,3 & 25,0 \\
\hline \multicolumn{3}{|l|}{ Reptilia: Iguanidae } \\
\hline Liolaemus sp. & 16,7 & \\
\hline \multicolumn{3}{|l|}{ Amphibia: Rhinodermatinae } \\
\hline Rhinoderma darwinii & 25,0 & \\
\hline Unidentified anuran & & 25,0 \\
\hline \multicolumn{3}{|l|}{ Insecta: Coleoptera } \\
\hline Carabidae: Ceroglosus chilensis & 16,7 & \\
\hline Carabidae: Creobius eudoxi & 16,7 & \\
\hline Elateridae: unidentified & 33,3 & 25,0 \\
\hline Lucanidae: unidentified & 25,0 & \\
\hline Scarabaeidae: Chiasognathus grantii & 66,7 & 12,5 \\
\hline Scarabaeidae: unidentified & 16,7 & \\
\hline \multicolumn{3}{|l|}{ Insecta: Diptera } \\
\hline Tipulidae: Tipula sp. & & 25,0 \\
\hline Tabanidae: Dasybasis sp. & 33,3 & \\
\hline Mycetophylidae: unidentified & 16,7 & \\
\hline \multicolumn{3}{|l|}{ Insecta: Lepidoptera } \\
\hline Heplalidae: unidentified & & 12,5 \\
\hline Arachnida: Aranae: unidentified & 16,7 & \\
\hline Annelida: Oligochaeta: unidentified & 33,3 & 12,5 \\
\hline Myriapoda: Geophillidae: unidentified & 8,3 & \\
\hline
\end{tabular}


TABLA 3

Media y rango de ítemes vegetales y animales expresados como porcentajes volumétricos del contenido estomacal de jabalíes silvestres en dos localidades de Chile

Mean and range of plant and animal items as percent volume by locality in stomach contents of wild boar in Chile

\begin{tabular}{|c|c|c|c|c|c|}
\hline $\begin{array}{l}\text { Ítemes vegetales } \\
\text { y animales }\end{array}$ & $\begin{array}{l}\text { Volcán Mocho- } \\
\text { Choshuenco } \\
(\mathrm{n}=12)\end{array}$ & $\begin{array}{l}\text { Parque Nacional } \\
\text { Vicente Pérez Rosales } \\
(\mathrm{n}=8)\end{array}$ & Valor de $\mathrm{t}(\mathrm{gl})$ & Valor de $\mathrm{P}$ & $\begin{array}{l}\text { Media anual } \\
\quad(\mathrm{n}=20)\end{array}$ \\
\hline Vegetales & $84,2(0,5-99,7)$ & $73,8(17,9-96,5)$ & $0,745(18)$ & 0,466 & 83,9 \\
\hline Gunnera tinctoria $*$ & $14,2^{\mathrm{a}}(3,6-52,4)$ & $43,6^{\mathrm{b}}(5,6-82,6)$ & $3,146(8)$ & 0,006 & 15,2 \\
\hline Otros vegetales & $39,7(0,5-83,6)$ & $24,2(12,3-59,4)$ & $1,438(18)$ & 0,168 & 42,0 \\
\hline Hongos * & $30,3^{\mathrm{a}}(0,5-76,5)$ & $6,1^{\mathrm{b}}(0,1-12,2)$ & $3,253(11)$ & 0,007 & 15,9 \\
\hline Animales & $15,7(0,1-29,5)$ & $25,8(3,5-81,1)$ & $1,055(8)$ & 0,333 & 16,1 \\
\hline Vertebrados * & $0,2^{\mathrm{a}}(0,1-1,5)$ & $24,8^{\mathrm{b}}(3,2-81,1)$ & $2,654(7)$ & 0,024 & 8.7 \\
\hline Invertebrados * & $15,5^{\mathrm{a}}(0,1-60,3)$ & $1,0^{\mathrm{b}}(0,1-2,8)$ & $2,495(11)$ & 0,030 & 7,4 \\
\hline
\end{tabular}

* Letras diferentes en superíndice indican diferencias significativas entre columnas para $\mathrm{P}<0,05$

En las muestras de PNVPR se encontró alta cantidad de restos de roedores tales como pelos, trozos de piel, garras, dientes y colas enteras (Tabla 3). En dos estómagos de jabalí subadultos se detectaron roedores enteros: una muestra presentaba los cuerpos casi intactos de tres especímenes y en siete de los ocho estómagos se encontró tricobezoares de 1-3 cm diámetro. Los tricobezoares son bolas de pelos, que indican que los jabalíes examinados llevaban algún tiempo comiendo ratones. La presencia de aves se determinó por el hallazgo de plumas, ranfoteca y garras. Restos de aves se encontraron en muestras de tres machos adultos y tres juveniles así como en dos hembras adultas $\mathrm{y}$ dos juveniles. En el contenido estomacal de una jabalina adulta del PNVPR en invierno, se estableció la presencia simultánea de Scelorchilus rubecula y Pteroptochos tarnii. Ejemplares de Amphibia aparecieron en cinco de las 20 muestras analizadas.

En relación con Insecta, las larvas constituyen un ítem frecuente en ambos sitios, representados por los mismos órdenes. El coleóptero Chiasognathus grantii constituye la mayor parte de los ejemplares larvales de insectos encontrados en $\mathrm{VMCH}$, llegando a extremos de 200 larvas por estómago (Tabla 3). En el caso de las muestras del PNVPR, solo en un estómago se encontró larvas de $C$. grantii. Annelida aparece en cinco muestras y el consumo de lombrices probablemente constituye un ítem más frecuente, solo que la pesquisa de sus restos en el contenido estomacal es difícil debido a su fragilidad.

\section{Representación de ítemes vegetales y animales en las dos localidades}

Las cantidades relativas de los componentes de contenido estomacal según localidad de las muestras se presentan en la Tabla 3. Debido a la gran cantidad de rizoma de la nalca Gunnera tinctoria, esta especie se presenta separada de los restantes vegetales. Los hongos también se incluyen separados de los demás vegetales. Respecto de G. tinctoria y hongos se establecen diferencias $(\mathrm{P}<0,05)$ de consumo entre localidades, siendo innegable la importancia de la nalca, tanto en frecuencia como en volumen entre los componentes vegetales, a excepción de una sola muestra (macho adulto, verano en VMCH) que presentó Mimulus luteus equivalente al 83,6\% del volumen. Los ítemes vertebrados e invertebrados presentaron diferencias $(\mathrm{P}<0,05)$ entre muestras de $\mathrm{VMCH}$ y PNVPR (Tabla 3 ).

No se encontró diferencia significativa entre composición volumétrica y sexo o edad (Tabla 4), aun cuando juveniles muestran mayor consumo de ítem animal y machos de $G$. tinctoria. 
TABLA 4

Media y error estándar de ítemes vegetales y animales expresados como porcentajes volumétricos del contenido estomacal de jabalíes silvestres de Chile según sexo y edad

Mean and standard errors of plant and animal items as percent volume in stomach contents according to sex and age of wild boars in Chile

\begin{tabular}{|c|c|c|c|c|c|c|c|c|}
\hline \multirow{2}{*}{$\begin{array}{l}\text { Ítemes vegetales } \\
\text { y animales }\end{array}$} & \multicolumn{4}{|c|}{ Edad } & \multicolumn{4}{|c|}{ Sexo } \\
\hline & $\begin{array}{l}\text { Adultos } \\
(\mathrm{n}=15)\end{array}$ & $\begin{array}{c}\text { Juveniles } \\
(\mathrm{n}=8)\end{array}$ & $\begin{array}{l}\text { Valor de } \\
\mathrm{t}(\mathrm{gl})^{1}\end{array}$ & $\begin{array}{l}\text { Valor } \\
\text { de P }\end{array}$ & $\begin{array}{l}\text { Machos } \\
(\mathrm{n}=12)\end{array}$ & $\begin{array}{c}\text { Hembras } \\
(\mathrm{n}=8)\end{array}$ & $\begin{array}{l}\text { Valor de } \\
\text { t (gl) }\end{array}$ & $\begin{array}{l}\text { Valor } \\
\text { de P }\end{array}$ \\
\hline Vegetales & $84,1(6,4)$ & $67,8(12,6)$ & $1,227(18)$ & 0,236 & $77,2(9,5)$ & $84,4(3,3)$ & $0,718(13)$ & 0,477 \\
\hline Gunnera tinctoria & $27,3(6,5)$ & $18,3(8,0)$ & $0,739(18)$ & 0,470 & $30,0(8,3)$ & $17,7(6,2)$ & $1,075(18)$ & 0,297 \\
\hline Hongos & $22,5(5,8)$ & $14,9(7,0)$ & $0,682(18)$ & 0,504 & $18,8(7,0)$ & $23,5(5,5)$ & $0,482(18)$ & 0,635 \\
\hline Otros vegetales & $33,3(6,2)$ & $34,1(8,7)$ & $0,067(18)$ & 0,947 & $30,6(6,5)$ & $44,0(6,0)$ & $1,419(18)$ & 0,173 \\
\hline Animales & $15,7(6,3)$ & $31,9(12,4)$ & $1,224(18)$ & 0,237 & $22,7(9,4)$ & $15,6(3,3)$ & $0,663(13)$ & 0,521 \\
\hline Vertebrados & $4,9(2,2)$ & $25,5(14,9)$ & $1,368(4)$ & 0,252 & $10,9(6,7)$ & $7,5(3,9)$ & $0,427(16)$ & 0,681 \\
\hline Invertebrados & $10,1(3,9)$ & $8,4(4,3)$ & $0,238(18)$ & 0,815 & $10,1(4,9)$ & $9,0(2,7)$ & $0,914(16)$ & 0,850 \\
\hline
\end{tabular}

La agrupación de los ítemes vegetales y animales de acuerdo a si se encuentran normalmente sobre o bajo el suelo indica que, en muestras de invierno predominan $(70,8 \%$ vol) especies vegetales (rizoma de nalca) y animales (roedores y larvas de insecto) que viven o se desarrollan bajo el suelo. La particularidad del jabalí de hozar extensa y profundamente, le permiten acceder a un recurso trófico al cual tienen acceso solo de forma restringida algunos micromamíferos de la fauna nativa. En cambio, muestras de otoño, primavera y verano contenían en promedio 77,5 $\%$ vol de componentes epigeos. Respecto del consumo estacional, se detectaron diferencias $(\mathrm{P}<0,05)$ solo en consumo de quilas entre otoño e invierno. Para detectar diferencias entre primavera y verano, no existió suficiente poder de prueba con solo dos muestras $u$ observaciones por época.

\section{DISCUSIÓN}

Tal como se esperaba, la mayor parte de los ítemes identificados en los contenidos estomacales corresponde a especies endémicas o sudamericanas y no han sido descritas en la literatura como parte de la dieta de jabalí, lo que confirma el carácter generalista y oportunista de esta especie exótica (Oloff 1951, Briedermann 1986, Genov 1981a, 1981c,
Schley \& Roper 2003, Baubet et al. 2004). Las proporciones de ocurrencia y volumen de ítemes vegetales (100 y 80\% respectivamente) reflejan el carácter eminentemente vegetariano de la dieta de jabalí en el área, en concordancia con valores de 85,8 a 92,6 \% observados en jabalíes europeos (Janda 1958, Briedermann 1976, Genov 1981b). Gramíneas, hongos y Gunnera constituyen ítemes que predominan en muestras de primavera, otoño e invierno respectivamente, sugiriendo que el jabalí es capaz de concentrarse en unos pocos substratos o cambiar totalmente de dieta según disponibilidad (Scott \& Pelton 1975, Everitt \& Alaniz 1980, Wood \& Roark 1980, Howe et al. 1981, Baber \& Coblentz 1987, Dardaillon 1987, Groot Bruinderink et al. 1994, Asahi 1995, Fournier-Chambrillon et al. 1995, Sáenz de Buruaga 1995, Massei et al. 1997, Baubet et al. 2003, Herrero et al. 2004).

El jabalí se encuentra en Chile en forma silvestre, en el área boscosa andina desde el volcán Tolhuaca $\left(38^{\circ} 13^{\prime} \mathrm{S}, 71^{\circ} 43^{\prime} \mathrm{O}\right)$ en la IX Región hasta Lago Verde en la XI Región $\left(44^{\circ} 23^{\prime}\right.$ S, $72^{\circ} 03^{\prime}$ O) (Skewes 1990). Excluyendo aquellas áreas y ambientes donde no habita el jabalí (cordillera de la Costa y tipos forestales Lenga, Ciprés de las Guaitecas y Esclerófilo) y de acuerdo a datos del Catastro de Bosque Nativo (CONAF, CONAMA, BIRF 1999), su área de distribución comprende cerca de 1.350 .00 ha dominadas $(66,4 \%)$ por 
bosques del tipo Siempreverde (21,8 \%), Roble-Raulí-Coihue $(20,5 \%)$ y Coihue-RaulíTepa $(24,1 \%)$. Estos tipos forestales destacan en el área de muestreo del Volcán MochoChoshuenco (VMCH) con Roble-Raulí-Coihue y Coihue-Raulí-Tepa y en el área de Parque Nacional Vicente Pérez Rosales (PNVPR) con Siempreverde y Coihue-Raulí-Tepa. En consecuencia, nuestros resultados provienen de aquellos hábitats de mayor representación en la distribución nacional de jabalí. Las hojas registradas de Nothofagus dombeyi eran en su mayoría de color pardo, como las que se encuentran en la hojarasca del suelo del bosque. Por lo tanto no deberían considerarse como parte de la dieta, sino más bien como acompañantes incidentales resultantes de la actividad de remoción edáfica del jabalí.

Las diferencias $(\mathrm{P}<0,05)$ detectadas entre VMCH y PNVPR para volumen de hongos en la dieta pueden reflejar cambios en la disponibilidad en terreno. El volumen consumido de $G$. tinctoria es mayor $(\mathrm{P}<0,05)$ en PNVPR, pero no si el análisis se restringe a muestras de invierno siendo el ítem vegetal más consumido en esta época. Al comparar el valor estimado de energía digestible de $17,1 \mathrm{MJ} \mathrm{kg}^{-1}$ MS del rizoma de nalca con alimentos utilizados en alimentación de cerdos (Sus scrofa) como avena (Avena spp.) que aporta 12,6 $\mathrm{MJ} \mathrm{kg}^{-1}$ y maíz (Zea maiz) con 16,0 MJ $\mathrm{kg}^{-1}$ (National Research Council 1998) resulta que $G$. tinctoria es un ítem alimentario de valor energético superior a ambos. Además, la fibra bruta está inversamente relacionada con la digestibilidad (Noblet \& Pérez 1993) y el bajo valor relativo de FB de nalca $(10,8 \%)$ podría explicar los altos niveles de consumo por parte del jabalí. No obstante, es interesante notar que el rizoma tiene un gran contenido de taninos (Campos 1999), compuestos que tienen la capacidad de, una vez en el intestino de vertebrados, unirse a proteínas reduciendo su disponibilidad y digestibilidad y por tanto la energía disponible para el animal (Magnione \& Bozinovic 2003).

La alta proporción en la dieta de vegetales que crecen dentro del bosque, concuerda con el comportamiento eminentemente boscoso del jabalí en su tierra típica (Genov 1981b, Henning 2001, Virgos 2002). El predominio estacional (invierno) de actividad edáfica del jabalí, es afín con observaciones reportadas en otros estudios (Genov 1981b, Dardaillon 1987, Kotanen 1995, Welander 2000, Herrero et al. 2004). La alta frecuencia de ocurrencia de brácteas de Chusquea, una gramínea bambúsea, en los estómagos evidencia una ligazón del jabalí al ambiente de Chusquea. Respecto a las gramíneas en la dieta, se señala que el jabalí tiene una mayor capacidad para digerir la fibra detergente neutra (FDN) en comparación con el cerdo doméstico y que la FDN podría proveer $26 \%$ de la energía digerible y que el pasto fresco podría considerarse un alimento de primera necesidad para la especie (Van Wieren 2000). Cerdos asilvestrados en Estados Unidos de América también consumen gramíneas preferentemente en estados fenológicos tempranos (Baber \& Coblentz 1987).

Los cuerpos fructíferos de hongos aparecen en alta ocurrencia y con una cifra muy similar al $69,5 \%$ reportado para cerdos asilvestrados en Estados Unidos de América (Wood \& Roark 1980). Los hongos no son un ítem importante en la dieta en Europa, con valores de 3 a $32 \%$ de ocurrencia, excepto en años de pobre semillación de Fagus y Quercus o cuando los animales no son forrajeados artificialmente (Briederman, 1976, Genov 1981c, Groot Bruinderink et al. 1994, Sáenz de Buruaga 1995, Baubet et al. 2004). En nuestro estudio se detectó un máximo de ocurrencia en otoño, mientras que en Europa es en invierno (Genov 1981b, Herrero et al. 2004). La micofagia es habitual en micromamíferos de bosques del hemisferio norte (Ovasaka \& Herman 1986, Meyer et al. 2005) como de bosques de Nothofagus en Sudamérica (Pearson \& Pearson 1982, Meserve et al 1988). Los hongos son relativamente pobres nutritivamente (Fogel \& Trappe 1978, Groenwall \& Pehrson 1984) pero son importantes en la dieta de los micromamíferos debido a su abundancia y fácil acceso (Cork \& Kenagy 1989). Las hojas del helecho Blechnum chilense se detectaron en invierno en muestras del PNVPR. Rizomas de helechos también son parte de la dieta de jabalí en Europa (Briedermann 1976) y de cerdos asilvestrados en Estados Unidos de América (Bratton 1975).

La alta ocurrencia de ítemes animales (100 $\%$ ) concuerda con registros para Europa donde el componente animal está siempre presente en la dieta de jabalí (Schley \& Roper 2003 ). No obstante la proporción volumétrica de 16,1\% 
de materia animal en nuestro estudio, está por sobre los valores reportados de 7-13\% para Europa (Janda 1958, Briedermann 1976, Groot Bruinderink et al. 1994, Sáenz de Buruaga 1995) y de $6 \%$ en Estados Unidos de América (Henry \& Conley 1972). El alto porcentaje en nuestro estudio se explica por el gran aporte de roedores en muestras de PNVPR (24,8\%), que tiene su origen en las denominadas "ratadas". Las ratadas consisten en un aumento explosivo de tamaño poblacional de roedores sigmodontinos en parte como respuesta a la semillación de Chusquea (Mann 1978, Gallardo \& Mercado 1999, Jaksic \& Lima 2003). Este hecho aconteció en PNVPR durante la temporada estival precedente a la recolección de muestras de jabalí. González et al. (2000) reportaron para el área incrementos poblacionales de 10 a 15 veces, para Oligoryzomys longicaudatus (422 \pm 33 individuos ha $\left.{ }^{-1}\right)$ y Abrothrix olivaceus (337 \pm 28 individuos $\mathrm{ha}^{-1}$ ) en fecha coincidente con la toma de muestras de nuestro estudio. Esto confirma el carácter oportunista del jabalí (Dardaillon 1987), que aprovechó la abundancia del ítem roedores. En una muestra de VMCH se registró Geoxus valdivianus, un roedor insectívoro de hábito semifosorial de los bosques templados de Chile (Meserve \& Jaksic 1991, Murúa 1995). Los micromamíferos subterráneos son un alimento preferido de jabalí en Europa (Oloff 1951, Bobak 1957, Schneider 1975, Briedermann 1976), incluyendo crías de conejos (Oryctolagus cuniculus, Sáenz de Buruaga 1995), que obtiene hozando sus respectivas cuevas.

La alta frecuencia de ocurrencia en estómagos (45 \%) de aves de la familia Rhinocryptidae llama la atención, en particular en muestras de PNVPR (63\%). Scelorchilus rubecula y Pteroptochos tarnii son especies endémicas y relativamente abundantes, con existencia terrícola en el sotobosque de Nothofagus y dentro de densos matorrales de Chusquea (Correa et al. 1990, Jaksic \& Feinsinger 1991, Reid et al. 2004). En Europa el jabalí también depreda sobre aves, en especial sobre huevos y crías de aves que nidifican en el suelo (Henry 1969, Henry \& Conley 1972, Scott \& Pelton 1975, Herrero et al. 2004) y es sindicado como responsable de la baja poblacional de la becasina (Scolopax rusticola) en bosques europeos (Nyenhuis
1998). El ítem Amphibia aparece en $25 \%$ de los estómagos examinados. Debido a la fragilidad de los cadáveres de Amphibia, es difícil detectar sus restos en el contenido estomacal y por tanto la frecuencia de estas especies consumidas por el jabalí en Chile podría ser mayor. La frecuencia de batracios en la dieta de jabalí en Europa es variable, con valores de 3 a $40 \%$ y restringido al invierno (Briedermann 1986). En Australia, el consumo de batracios es más alto (Tisdell 1984, Lever 1985).

Los porcentajes de ocurrencia $(70 \%)$ de la clase Insecta en el presente estudio se ubican en el tercio superior respecto de valores en la dieta de jabalí en el hemisferio norte (Scott \& Pelton 1975, Briedermann 1976, Wood \& Roark 1980, Genov 1981b, Howe et al. 1981). El jabalí es un efectivo controlador de plagas de insectos forestales cuyas larvas habitan el suelo (Genov 1981c, Briederman 1986). En nuestro estudio, la mayor representación por ocurrencia está dada por Coleoptera, en especial larvas de Chiasognattus grantii (67\% en VMCH), que son parte importante de la dieta de micromamíferos de los bosques patagónicos de Nothofagus (Pearson \& Pearson 1982). Solo Sáenz de Buruaga (1995) en España, indica cierta predilección del jabalí por orugas de coleópteros. Respecto de Annelida, en varios estudios de dieta de jabalí (Challies 1975, Scott \& Pelton 1975, Howe et al. 1981, Baubet et al. 2004) el ítem lombrices de tierra es más alto y constante que en el nuestro, llegando en algunos casos a ser el ítem animal más importante de la dieta (Challies 1975, Asahi 1995).

La composición del contenido estomacal no presentó diferencias entre machos y hembras, al igual que en Europa (Groot Bruinderink et al 1994, Schley \& Roper 2003) y en cerdos asilvestrados de Norteamérica (Wood \& Roark 1980). Respecto de edad, si bien los individuos juveniles contenían mayor volumen de ítem animal, no hubo diferencia estadística. En Europa, los jabalíes juveniles consumen mayor proporción de ítem animal (Dardaillon 1989, Groot Bruinderink et al. 1994, Labudzki \& Wlazelko 1998,) debido a su mayor demanda proteica para sostener el crecimiento (Dardaillon 1989).

En conclusión, la dieta del jabalí silvestre en Chile centro-sur está representada por animales, plantas y hongos. La caracterización de esta 
dieta permite situar al jabalí como una especie generalista y oportunista cuyo impacto sobre la flora y fauna nativa requiere ser evaluado.

\section{AGRADECIMIENTOS}

A los revisores anónimos y a Luis Ebensperger editor de la revista, por sus valiosas sugerencias. A Nolberto Poblete, por su acogida y enseñanza en terreno. A CONAF X Región, Andina del Sud y Peña de Caza Los Guarros, por su ayuda en la obtención de muestras.

\section{LITERATURA CITADA}

ALEXIOU PN (1983) Effect of feral pigs (Sus scrofa) on subalpine vegetation at Smokers Gap. Proceedings of the Ecological Society of Australia 12: 135-142.

ANÓNIMO (1956) Schwarzwild in Argentinien. Wild und Hund 59: 12.

AOAC (1997) Association of Official Analytical Chemists Official Methods of Analysis (15th Ed.), P. Cuniff., Gaithesburg, Maryland USA.1298 pp.

ARMESTO JJ ,C VILLAGRAN \& MK ARROYO (1997) Ecología de los bosques nativos de Chile. Editorial Universitaria, Santiago, Chile. 477 pp.

ASAHI M (1995) Stomach contents of Japanese wild boar in winter. Ibex, Journal of Mountain Ecology 3: 184-185.

BABER DW \& BE COBLENTZ (1987) Diet, nutrition and conception in feral pigs on Santa Catalina Island. Journal of Wildlife Management 51: 306-317.

BARRET RH (1977) Wild pigs in California. En: Wood GW (ed) Research and management of wild hog populations: 111-113. Belle W. Baruch Forest Science Institute of Clemson University, Georgetown, South Carolina, USA.

BAUBET E, Y ROPERT-COUDERT \& S BRANDT (2003) Seasonal and annual variations in earthworm consumption by wild boar (Sus scrofa scrofa L.). Wildlife Research 30: 179-186.

BAUBET E, C BONENFANT \& S BRANDT (2004) Diet of the wild boar in the French Alps. En: Fonseca C, J Herrero, A Luís \& AMVM Soares (eds) Wild boar research 2002: a selection of edited papers from the $4^{\text {Th }}$ International Wild Boar Symposium: 101-113. Sociedad Española para la Conservación y Estudio de los Mamíferos (SECEM) Málaga, España.

BIEBER C \& T RUF (2005) Population dynamics in wild boar Sus scrofa: ecology, elasticity of growth rate and implications for the management of pulsed resource consumers. Journal of Applied Ecology 42: $1203-1213$

BOBACK AW (1957) Das Schwarzwild. Neumann Verlag, Germany. $142 \mathrm{pp}$.

BRATTON SP (1975) The effect of the European wild boar, Sus scrofa, on gray beech forest in the Great Smoky Mountains. Ecology 56: 1356-1366.

BRIEDERMANN L (1976) Ergebnisse einer Inhaltsanalyse von 665 Wildschweinmägen. Der Zoologische Garten 46: 157-185.
BRIEDERMANN L (1986) Schwarzwild. VEB Deutscher Landwirtschaftsverlag, Berlin, Eastern Germany. $539 \mathrm{pp}$.

CAMPBELL DJ \& MR RUDGE (1984) Vegetation changes induced over ten years by goats and pigs at Port Ross, Auckland Islands (Subantartic). New Zealand Journal of Ecology 7: 103-118

CAMPOS J (1999) Productos forestales no madereros en Chile. Serie Forestal 10 Corporación de Investigación Tecnológica, INTEC-CHILE y FAO, Santiago, Chile. 65pp.

CHALLIES CN (1975) Feral pigs (Sus scrofa) on Auckland Island: Status and effects on vegetation and nesting sea birds. New Zealand Journal of Zoology 2: 198-206.

COBLENTZ BE \& DW BABER (1987) Biology and control of feral pigs on Isla Santiago, Galapagos, Ecuador. Journal of Applied Ecology 24: 403-418.

CONAF, CONAMA, BIRF (1999) Universidad Austral de Chile, Pontificia Universidad Católica de Chile, Universidad Católica de Temuco. 1999. Catastro y Evaluación de los Recursos Vegetacionales Nativos de Chile. Informe Nacional con Variables Ambientales, Santiago, Chile. 88 pp.

CONSEJO FEDERAL DE INVERSIONES (1961) Serie evaluación de los recursos naturales de la Argentina (Primera Etapa), Tomo VIII Fauna Silvestre. Buenos Aires, Argentina.

CORK SJ \& KENAGY GJ (1989) Nutritional value of hypogeous fungus for a forest-dwelling ground squirrel. Ecology 70: 577-586.

CORREA A, JJ ARMESTO, R SCHLATTER, R ROZZI \& JC TORRES-MURA (1990) La dieta del chucao (Scelorchilus rubecula), un passeriforme terrícola endémico del bosque templado húmedo de Sudamérica austral. Revista Chilena de Historia Natural 63: 197-202.

CRUZ FC, J DONLAN, K CAMPBELL \& V CARRION (2005) Conservation action, Galapagos: feral pig (Sus scrofa) erradication from Santiago Island. Biological Conservation 121: 473-478.

DACIUK J (1978) Estado actual de las especies de mamíferos introducidos en la subregión Araucana (Rep. Argentina) y grado de coacción ejercido en algunos ecosistemas surcordilleranos. Anales de Parques Nacionales (Argentina) 14: 105-130.

DARDAILLON M (1987) Seasonal feeding habits of the wild boar in a mediterranean wetland, the Camargue (southern France). Acta Theriologica 32: 389-401.

DARDAILLON M (1989) Age-class influences on feeding choices of free-ranging wild boars (Sus scrofa). Canadian Journal of Zoology 47: 2792-2796.

DONOSO C (1981) Tipos forestales de Chile. Documento de Trabajo 38, Investigación y Desarrollo Forestal, Publicación CONAF, PNUD y FAO), Santiago, Chile. $70 \mathrm{pp}$

DONOSO C (1993) Bosques templados de Chile y Argentina. Editorial Universitaria, Santiago, Chile. $484 \mathrm{pp}$.

EVERITT JH \& MA ALANIZ (1980) Fall and winter diets of feral pigs in south Texas. Journal of Range Management 33: 126-129.

FOGEL R \& JM TRAPPE (1978) Fungus consumption (Mycophagy) by small mammals. Northwest Science 52: 1-30.

FOURNIER-CHAMBRILLON C, D MAILLARD \& P FOURNIER (1995) Diet of the wild boar (Sus scrofa L.) inhabiting the Montpellier garrigue. Ibex, Journal of Mountain Ecology 3: 174-179. 
GALLARDO MH \& C MERCADO (1999) Mast seeding of bamboo shrubs and mouse outbreaks in southern Chile. Mastozoología Neotropical (Argentina) 6: 103-111.

GARRIDO N (1988) Agaricales s.l. und ihre Mikorrhizen in den Nothofagius-Wäldern Mittelchiles. Bibliotheca Mycoologica. J. Cramer Verlagsbuchhandlung, Berlin, Germany, 528 pp.

GENOV P (1981a) Die Verbreitung des Schwarzwildes (Sus scrofa) in Eurasien und seine Anpassung an die Nahrungsverhältnisse. Zeitschrift für Jagdwissenschaft 27: 221-231.

GENOV P (1981b) Food consumption of wild boar in north-eastern and western Poland. Acta Theriologica 26: 185-205.

GENOV P (1981c) Significance of natural biocenoses and agrocenoses as the source of food for wild boar (Sus scrofa). Ekologia Polska 29: 117-136.

GONZÁLEZ LA, R MURÚA \& C JOFRÉ (2000) Uso del hábitat por dos especies de múridos en respuesta a un aumento poblacional (ratada) en los bosques templados del sur de Chile. Revista Chilena de Historia Natural 73: 489-495.

GROENWALL O \& A PEHRSON (1984) Nutrient content in fungi as a primary food of the red squirrel Sciurus vulgaris L. Oecologia (Berlin) 64: 231-231.

GROOT BRUINDERINK GW, E HAZEBROEK \& H VAN DER VOOT (1994) Diet and condition of wild boar, Sus scrofa scrofa, without supplementary feeding. Journal of Zoology (London) 233: 631-648.

HAJEK ER \& F DI CASTRI (1975) Bioclimatografía de Chile. Dirección de Investigación, Vicerrectoría Académica, Universidad Católica de Chile, Santiago, Chile. 107 pp.

HECK L \& G RASCHKE (1985) Die Wildsauen. Verlag P. Parey, Hamburg \& Berlin, 2 Auflage, 223 pp.

HENNING R (2001) Schwarzwild, Biologie, Verhalten, Hege und Jagd. BLV Verlag, München, Germany. $271 \mathrm{pp}$

HENRY VG (1969) Predation on dummy nests of groundnesting birds in the southern Appalachians. Journal of Wildlife Management 33: 169-172.

HENRY VG \& RH CONLEY (1972) Fall foods of the European wild hogs in the southern Appalachians. Journal of Wildlife Management 36: 854-860.

HERRERO J, S COUTO, C ROSELL \& P ARIAS (2004) Preliminary data on the diet of wild boar living in a Mediterranean costal wetland. En: Fonseca C, J Herrero, A Luís \& AMVM Soares (eds) Wild boar research 2002: 115-123. Sociedad Española para la Conservación y Estudio de los Mamíferos (SECEM) Málaga, España.

HERRERO J, A GARCÍA-SERRANO, S COUTO, V ORTUÑO \& R GARCÍA-GONZÁLEZ (2006) Diet of wild boar Sus scrofa L. and crop damage in an intensive agroecosystem. European Journal of Wildlife Research 52: 245-250.

HOWARD WE (1964) Introduced browsing mammals and habitat stability in New Zealand. Journal of Wildlife Management 28: 421-429.

HOWE TD, FJ SINGER \& BB ACKERMAN (1981) Forage relationships of European wild boar invading northern hardwood forests. Journal of Wildlife Management 45: 748-754

JANDA M (1958) Die Nahrung des Scharzwildes (Sus srofa) im Mittelgebirge von Stavnica. Säugetierkundliche Mitteilungen 6: 67-74.

JAKSIC FM (1998) Vertebrate invaders and their ecological impacts in Chile. Biodiversity \& Conservation 7: 1427-1445.
JAKSIC FM \& P FEINSINGER (1991) Bird assemblages in temperate forests of North and South America: a comparison of diversity, dynamics, guild structure, and resource use. Revista Chilena de Historia Natural 64: 491-510.

JAKSIC FM \& M LIMA (2003) Myths and facts on ratadas: bamboo blooms, rainfall peaks and rodent outbreaks in South America. Austral Ecology 28: 237-251

JAKSIC FM, JA IRIARTE, JE JIMÉNEZ \& DR MARTÍNEZ (2002) Invaders without frontiers: cross-border invasions of exotic mammals. Biological Invasions 4: 157-173.

KOTANEN PM (1995) Responses of vegetation to a changing regime of disturbance: effects of feral pigs in a Californian coastal prairie. Ecography 18: 190-199.

LABUDZKI L \& M WLAZELKO (1998) Nahrungskomponenten und trophische Stellung des Schwarzwildes im Forschungsgebiet Zielonka. En: Commichau C \& H Sprnakel (eds) Symposium zur Ökologie des Schwarzwildes: 83-90. Arbeitskreis Wildbiologie an der Justus-Liebeig-Universität Giessen, Germany.

LEVER C (1985) Naturalized mammals of the world Longman Press, London, United Kingdom. 487 pp.

LEVER C (1994) Naturalized animals. Cambridge University Press, Cambridge, United Kingdom. 354 pp.

LONG J (2003) Introduced mammals of the world. Their history, distribution and influence. CSIRO Publishing, Collingwood, Australia. 589 pp.

MACKIN R (1970) Dynamics of damage caused by wild boar to different agriculture crops. Acta Theriologica 15: 447-458.

MAGNIONE AM \& F BOZINOVIC (2003) Ecología nutricional y estrategias de digestión: Compromisos entre obtención de energía y eliminación de toxinas. En: Bozinovic F (Ed) Fisiología ecológica \& evolutiva: 125-150. Ediciones Universidad Católica de Chile, Santiago, Chile.

MANN G (1973) Mamíferos y aves introducidos a Chile. Corporación Nacional Forestal, Santiago, Chile, Publicación $\mathrm{N}^{\circ} 8,5 \mathrm{pp}$

MANN G (1978) Los pequeños mamíferos de Chile: marsupiales, quirópteros, edentados y roedores. Gayana Zoología (Chile) 40: 1-342.

MARTICORENA C \& M QUEZADA (1985) Catálogo de la flora vascular de Chile. Gayana Botánica (Chile) 42: 1-157.

MASSEI G, PV GENOV, BW STAINES \& ML GORMAN (1997) Factors influencing home range and activity of wild boar (Sus scrofa) in a Mediterranean coastal area. Journal of Zoology (London) 242: 411-423.

MATSCKE GH (1967) Aging European wild hogs by dentition. Journal of Wildlife Management 31: 109113.

McDONALD P, R EDWARDS \& JFD GREENHALGH (1993) Nutrición animal. Cuarta edición. Editorial Acribia, Zaragoza, España. 571 pp.

MELLA JE, JA SIMONETTI, AE SPOTORNO \& LC CONTRERAS (2002) Mamíferos de Chile. En: Ceballos G \& JA Simonetti (eds) Diversidad y conservación de los mamíferos neotropicales: 151183. CONABIO-UNAM, México, Distrito Federal, México.

MESERVE PL, BK LANG \& BD PATTERSON (1988) Tropical relationships of small mammals in a Chilean temperate rainforest. Journal of Mammalogy 69: 721-730. 
MESERVE PL \& FM JAKSIC (1991) Comparaciones de ensambles de vertebrados terrestres de pluviselvas templadas de Norte y Sudamérica. Revista Chilena de Historia Natural 64: 511-536.

MEYER M D, NORTH M P \& DA KELT (2005) Fungi in the diets of northern flying squirrels and lodgepole chipmunks in the Sierra Nevada. Canadian Journal of Zoology 83: 1581-1589.

MILLER S \& J ROTTMANN (1976) Lista de mamíferos chilenos. Corporación Nacional Forestal, Departamento de Conservación del Medio Ambiente, Sección Vida Silvestre, Santiago, Chile, Mimeografiado. 7 pp.

MOONEY HA, RN MACK, JA McNEEL, LE NEVILLE, PJ SCHEI \& JKWAAGE (2005) Invasive alien species: a new synthesis. Island Press, Washington, District of Columbia, USA. 368 pp.

MURÚA R (1995) Comunidades de mamíferos del bosque templado de Chile. En: Armesto JJ, C Villagrán \& MTK Arroyo (eds) Ecología de los bosques nativos de Chile: 113-133. Editorial Universitaria, Santiago, Chile.

NATIONAL RESEARCH COUNCIL (1998) Nutrient requirements of swine. Tenth edition. National Academy Press, Washington, District of Columbia, USA. $189 \mathrm{pp}$.

NOBLET J \& JM PÉREZ (1993) Prediction of digestibility of nutrients and energy values of pigs diets from chemical analysis. Journal of Animal Science 71 3389-3398.

NYENHUIS H (1998) Schwarzwild (Sus scrofa) versus Waldschnepfe (Scolopax rusticola). En: Commichau C \& H Sprankel (eds) Symposium zur Ökologie des Schwarzwildes: 131-135. Arbeitskreis Wildbiologie an der Justus-Liebeig-Universität Giessen, Germany

OLOFF H-B (1951) Zur Biologie und Ökologie des Scharzwildes. Dr. P. Schöps Verlag, Germany. 95 pp.

OVASAKA K \& TB HERMAN (1986) Fungal consumption by six species of small mammals in Nova Scotia. Journal of Mammalogy 67: 208-211.

PEÑA LE (1988) Introducción a los insectos de Chile. Segunda edición. Editorial Universitaria, Santiago, Chile. 253 pp.

PEARSON OP \& AE PEARSON (1982) Ecology and biogeography of the southern rainforests of Argentina. En: Mares MA \& HH Genoways (eds) Mammalian biology in South America: 129-142. Special Publications Series, Pymatuning Laboratory of Ecology, University of Pittsburgh, Pittsburgh, Pennsylvania, USA

PERTRIDES GA (1975) Guideline principles to govern the importation of hoofed animals. Environmental Conservation 2: 133-135.

PINE RH, SD MILLER \& ML SCHAMBERGER (1979) Contributions to the mammalogy of Chile. Mammalia 43: 337-376.

REID S, I DÍAZ, JJARMESTO \& MFWILSON (2004) Importance of native bamboo for understory birds in Chilean temperate forests. Auk 121: 515-525.

SÁENZ DE BURUAGA M (1995) Alimentación del jabalí
(Sus scrofa castilianus) en el norte de España. Ecología 9: 367-386.

SCOTT CD \& MR PELTON (1975) Seasonal food habits of the European wild hog in the Great Smoky Mountains National Park. Proceedings of the Southeastern Association of Game and Fish Commissions 29: 585-593.

SCHLEY L \& TJ ROPER (2003) Diet of the wild boar (Sus scrofa) in western Europe, with particular reference to consumption of agricultural crops. Mammal Review 33: 43-56.

SCHNEIDER E (1975) Mäuse in Magen eines Wildschweines (Sus scrofa, L.) Zeitschrift für Jagdwissenschaft 21: 190-192.

SINGER FJ (1981) Wild pig populations in the national parks. Environmental Management 5: 263-270.

SKEWES O (1990) Status des Wildschweines, Sus scrofa L., in Chile. Dokumentation einer Fremdansiedlung von europäischem Wild. Dissertation Doktorgrades Forstwissenschaft Fakultät Georg-August Universität Göttingen, Germany. 248 pp.

TAMAYO M, H NÚÑEZ \& J YÁÑEZ (1987) Lista sistemática actualizada de los mamíferos vivientes en Chile y sus nombres comunes. Noticiario Mensual Museo Nacional de Historia Natural (Chile) 312: 1-12

TISDELL CA (1982) Wild pigs: environmental pest or economic resource? Pergamon Press, Rushcutters Bay, Australia, $455 \mathrm{pp}$.

TISDELL C.A (1984) Feral pigs threaten native wildlife in Australia. Tigerpaper 2: 13-18.

VAN WIEREN SE (2000) Digestibility and voluntary intake of roughages by wild boar and Meshian pigs. Animal Science 71: 149-156.

VEBLEN TT, DH ASHTON, S RUBIULIS, DC LORENZ \& M CORTEZ (1989) Nothofagus stand development on intransit moraines, Casa Pangue Glacier, Chile. Artic \& Alpine Research 21: 144155.

VIRGOS E (2002) Factors affecting wild boar (Sus scrofa) occurrence in highly fragmented Mediterranean landscapes . Canadian Journal of Zoology 80: 430435.

VITOUSEK PM, HA MOONEY, J LUBCHENCO \& JM MELILLO (1997) Human domination of Earths's ecosystems. Science 277: 494-499.

VTOROV IP (1993) Feral pig removal: effects on soil microarthropods in Hawaiian rain forest. Journal of Wildlife Management 57: 875-880.

WELANDER J (1995) Are wild boar a future threat to the Swedish flora? Ibex, Journal of Mountain Ecology 3: $165-167$

WELANDER J (2000) Spatial and temporal dynamics of wild boar (Sus scrofa) rooting in a mosaic landscape. Journal of Zoology (London) 252: 263-271

WODZICKI KA (1950) Introduced mammals of New Zealand. Department of Scientific and Industrial Research, Wellington, New Zealand, Bulletin 98: 1255

WOOD GW \& DN ROARK (1980) Food habits of feral hogs in coastal South Carolina. Journal of Wildlife Management 44: 506-511. 
